\title{
Two-channel of real time data acquisition based on VB
}

\author{
Bingyan Wang ${ }^{a}$, Manjie Guo ${ }^{b}$ \\ ${ }^{1}$ Baotou Vocational \& Technical College, Inner Mongolia 014030, China \\ ${ }^{2}$ Hebei University of Science and Technology, Hebei 050000, China \\ a1003764638@qq.com, bgmj_tg@163.com
}

Keywords: data reception; MSComm; serial communication.

\begin{abstract}
This paper describes two-channel real-time data acquisition, display and storage of the displacement. The measure and control software with VB has some functions, such as data receiving, real-time curve drawing, display and data storage using database. The receiving of the real-time data using serial communication. Exploiting offered by VB MSComm communication controlling part writes mouthfuls of communication procedure which can complete the data exchange between PC and one-chip computer. The real time curve has been drawn will be the use of two methods of line, using VB basic line drawing method approach to mapping.
\end{abstract}

\section{Introduction}

The collection of information and analog-to-digital conversion completed by data acquisition system will be send into computer for further calculation [1]. The kind of computer language to develop the measurement and control software needs to be considered. Microsoft has launched an object-oriented tool available for developing application, Visual Basic, which is user-friendly and high-efficiency [2-3].

\section{Serial communication}

MSComm control. Two methods are introduced here to develop the serial communication program using Visual Basic, one is MSComm control and another is WindowsAPI function. MSComm control is convenient to establish the connection with serial port when programming. It is one of ActiveX controls provided by Microsoft. Some important attributes are described.

(1) Comport. Set and return the number of communication port, and the default is COM1.

(2) Port Open. Set and return the state of communication port, and it is Boolean quantities.

(3) Setting. It is used to specify the data transfer rate, parity, data bits and stop bits.

(4) Input. Return and delete the flow of data in the receive buffer.

(5) InputLen Set and return the Input attribute and read the number of characters from the receiving area.

(6) Input Mode. Set or return the type of the received data.

(7) Output. Write the data flow to the transmit buffer.

(8) Threshold. Set and return the number of characters of buffer before the Oncomm event.

(9) Threshold. Set and return the smallest number of characters of buffer before the Oncomm event.

(10)OutBufferSize. Set and return the size of transmission buffer.

(11)InBufferSize. Set and return the size of receive buffer.

Establish channel. The PC will send the handshake signal's' to single chip microcomputer after initializing the serial port and clicking the starting button.

The single chip will send the response signal '0x23' after receiving the handshake signal's'. Communication will connect successfully when the PC receives the response signal. And then PC will send the command ' $t$ ' to inform the single chip to send data pre $50 \mathrm{~ms}$.

The flow of data interchange between PC and single chip is shown in figure 1. 


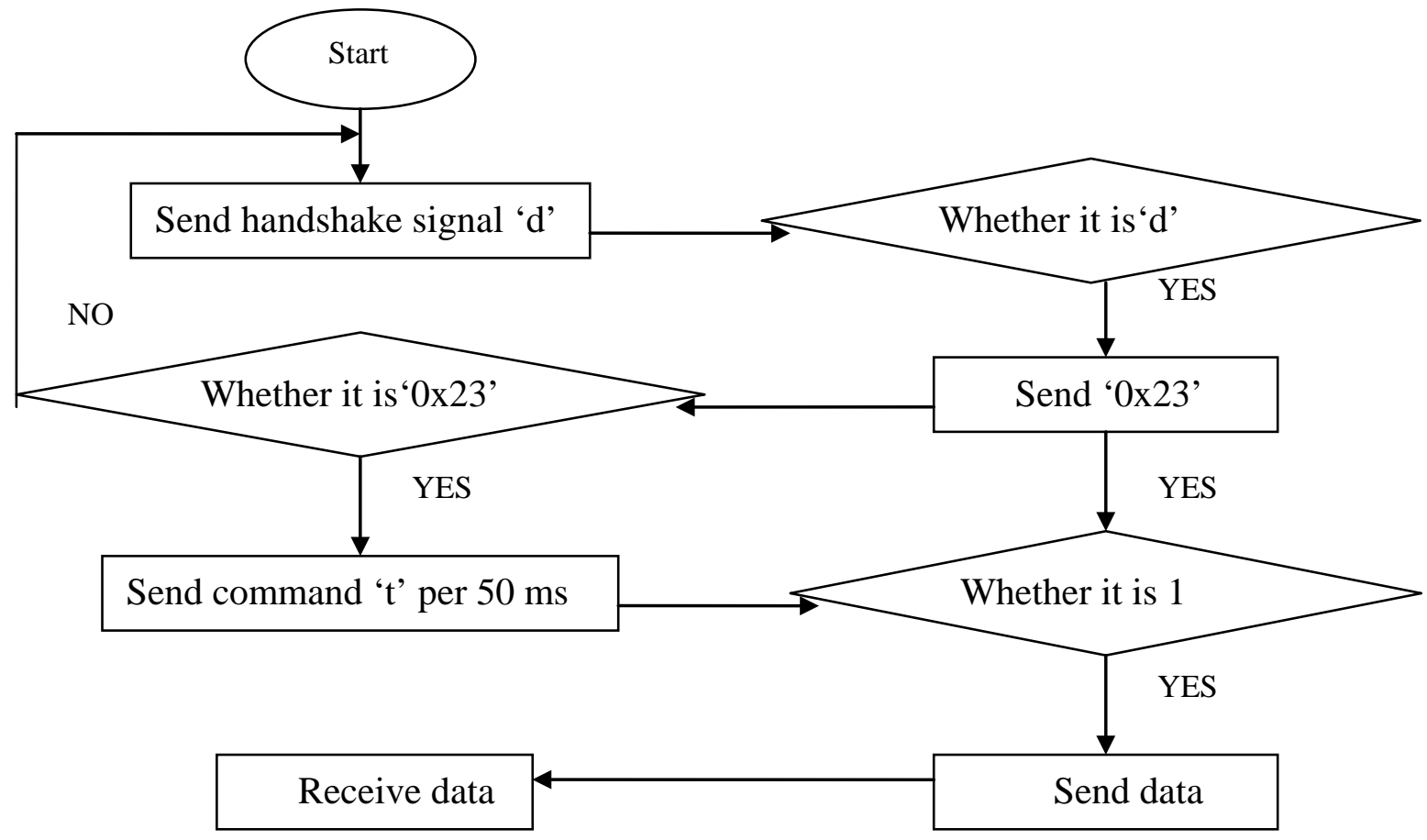

PC

Single chip microcomputer

Figure.1 Data Interchange Between PC and Single Chip

\section{System Design}

Data Reception. In order to ensure that the PC can receive the correct data, the format of frame is used to send data. The function in single chip has been shown below.

Void send char (void)

\{

unsigned char $\mathrm{i}=0$;

$\mathrm{C}[0]=0 \mathrm{xff}$;

$\mathrm{C}[1]=$ (uchar) press;

$C[2]=($ uchar $)$ (press $>>8)$;

$\mathrm{C}[3]=$ position;

$C$ [4] = (uchar) (press $>>8)$;

while $(\mathrm{i}<5)$

\{

SBUF $=\mathrm{c}[\mathrm{i}]$;

while(! TI);

$\mathrm{TI}=0$;

i++;

\}

\}

PC receives the data with the method of asynchronous. And the steps are conclude below.

Put the received data into an array of Inbyte.

Ensure the boundaries of superscript and subscript, and make $\mathrm{j}$ equals superscript, $\mathrm{i}$ equals subscript.

Judge whether flag1 is true, if it is yes then perform (6).

Judge Hex (Inbyte(i))='FF', if it is yes, then perform (5). 
$\mathrm{i}=\mathrm{i}+1$; judge $\mathrm{i}<=\mathrm{j}$, if it doesn't set up, the judge $\mathrm{m}=0$, if it is yes, then perform (1). Judge $m<4$, if it is not, then perform (8).

Buffer=buffer+Hex (Inbyte(i)) + chr(32); $m=m+1$; perform (5).

Call for function ConvertHexSin (buffer) to converse the data to decimal number. The flow is shown in figure 2.

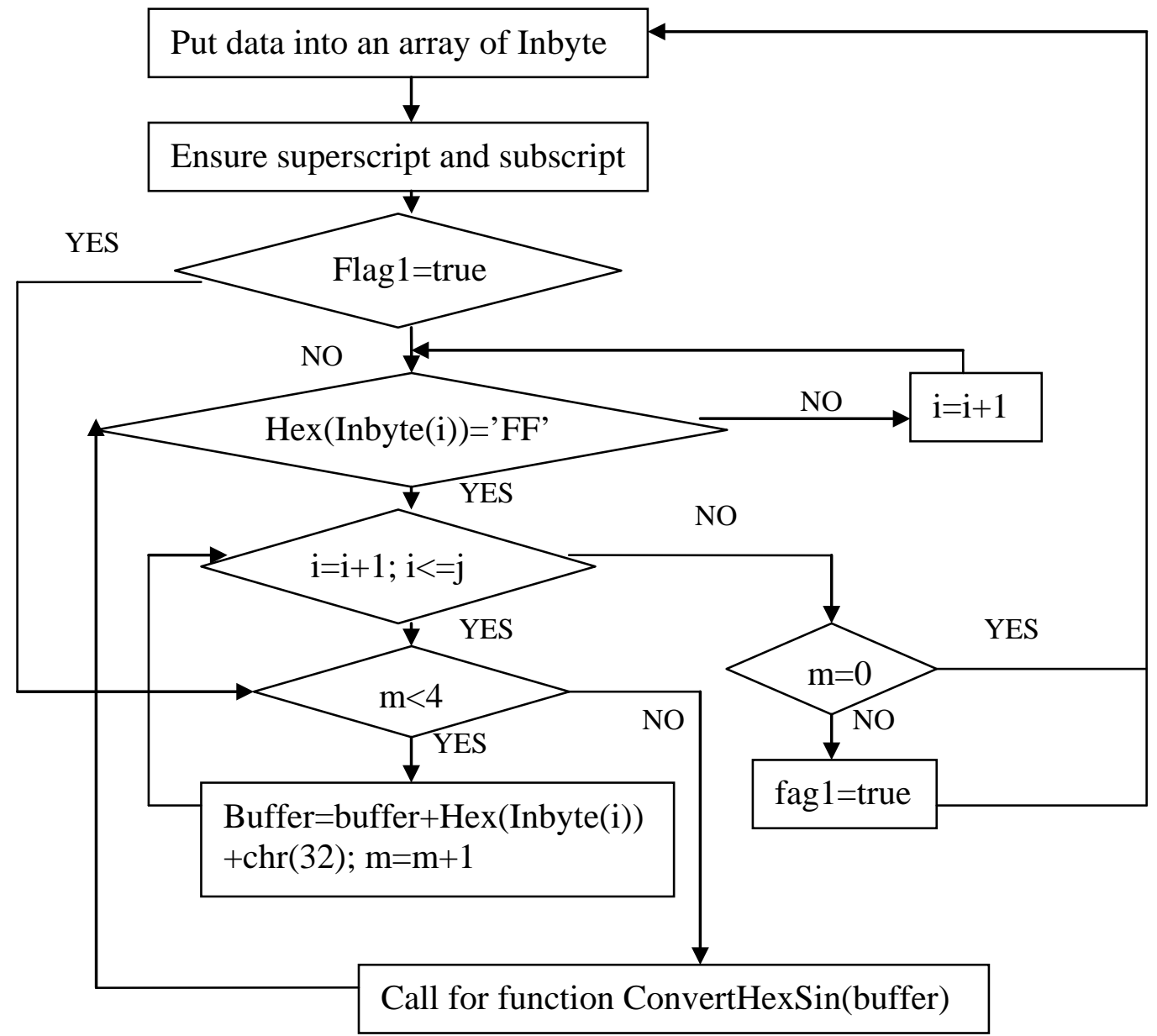

Figure.2 Flow of Data Producing

Testing Result. Figure 3 shows the two-channel real-time data acquisition of press and displacement.

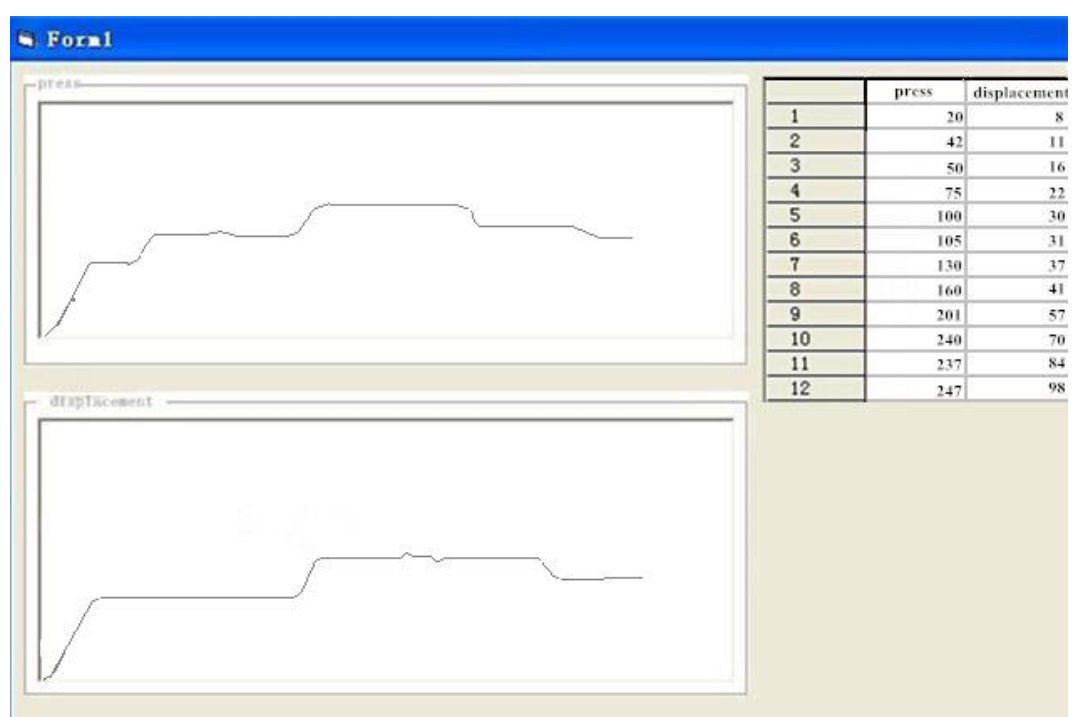

Figure.3 Testing Result 


\section{Summary}

This paper has proposed a method to describe two-channel real-time data acquisition. And Visual Basic has been used here as PC interface. A simple example has been illustrated here to verify the function of signals processing and graphic displaying accurately. The system provides information intuitively and has widely use.

\section{References}

[1] FAN Panguo, MA Dao-ping, WANG Guo-bin and HU Yixue. "Distributed Temperature Monitoring System Based on Single Chip Microcomputer”. Machinery \& Electronics, 07 (2013) p. 51-53.

[2] CHENG San-feng, LIU Xiao-bo. "Data-aquistion System Design based on Serial Communication between VB6.0 and SCM”. Automation \& Instrumentation, 01 (2004) p. 66-68.

[3] WANG Lu, XU Xuefeng, WANG Jian. “Application of Seral Communication in Energy monitoring system based on VB”. Journal of Nanchang Institute of Technology, 01 (2011) p. 33-37. 